\title{
Culture of Neospora caninum in the presence of a Mycoplasma Removal Agent results in the selection of a mutant population of tachyzoites
}

\author{
A. HUDSON and J. T. ELLIS* \\ Department of Cell and Molecular Biology, University of Technology, Sydney, St Leonard's Campus, Westbourne Street, \\ Gore Hill, NSW 2065, Australia
}

(Received 27 fuly 2004; revised 11 November 2004; accepted 15 November 2004)

S UMM A R Y

Mycoplasmas are common contaminants of eukaryotic cells grown in tissue culture. A commercially available Mycoplasma Removal Agent (MRA) was therefore assessed for its effect on tachyzoites of Neospora caninum, in order to determine its suitability for further use in parasite cell cultures. Analyses of tachyzoite and excreted-secreted proteins and antigens by SDS-PAGE and Western blotting show that MRA treatment results in the rapid selection of a mutant population that differs from the control and parental lines in its protein and antigen content. The treatment of $N$. caninum cultures with MRA is therefore not recommended for the eradication of Mycoplasma.

Key words: Neospora caninum, Mycoplasma, antibiotic, quinolone, culture.

\section{INTRODUCTION}

Mycoplasma infections are common contaminants of in vitro cultures of eukaryotic organisms, typically resulting in a reduction of cell growth and viability (Nakai et al. 2000; Uphoff \& Drexler, 2002). Current recommendations for the control of Mycoplasma are to discard the cultures or to treat with one of a number of antibiotics if the cultures are too valuable to throw away.

Although a symbioisis between Mycoplasma hominis and Trichomonas vaginalis is recognized (Rappelli et al. 2001), other infections by Mycoplasma of protozoal cultures are considered contaminants. Mycoplasma infections of cultures of Plasmodium were reported previously to be widespread (Turrini et al. 1997; Rowe et al. 1998), and clearly were of concern because of the ability of the bacterium to potentially alter the biological properties of the protozoans. Mycoplasma infections may also compromise a wide range of immunological studies because of their strong ability to induce tumour necrosis factor alpha (Hensmann \& Kwiatkowski, 2001). Furthermore Mycoplasma possesses an arginine deaminase, which is a highly expressed enzyme, involved in the metabolism of arginine to citrulline and ammonia (Misawa et al. 1994). There is therefore also the potential of Mycoplasma to interfere with studies on the metabolism of parasitic protozoa.

Most studies to date on the use of antibiotics to remove Mycoplasma from cell cultures have

\footnotetext{
* Corresponding author: Tel: +612 95144161. Fax: +61
}

2 95144003.E-mail: j.ellis@uts.edu.au concentrated on assaying features of cell viability and growth, and the phenomenon of drug resistance (Uphoff \& Drexler, 2004). The effects on parasitic protozoa have not been considered. In this study, we investigated the suitability of a commercially available Mycoplasma Removal Agent (MRA) for the treatment of in vitro cultures of Neospora caninum. Specifically we investigated the effects of the MRA treatment on the protein and antigen composition of tachyzoites grown in vitro.

\section{MATERIALS AND METHODS}

\section{Parasites}

Tachyzoites of $N$. caninum (Nc-Nowra or NcLiverpool strain) were grown on a monolayer of Vero cells as described previously (Miller et al. 2002) using RPMI 1640 medium supplemented with $2 \mathrm{~mm}$ glutamine, heat-inactivated horse serum (5-10\%) and $1 \%$ penicillin/streptomycin. Cultures were maintained at $37{ }^{\circ} \mathrm{C}$ in an atmosphere containing $5 \% \mathrm{CO}_{2}$.

\section{Treatment of cultures with Mycoplasma Removal Agent}

A single culture of Mycoplasma-free Nc-Nowra was divided into two at the time of normal passage. One half was treated for 14 days with MRA (MP Biomedicals Australasia Pty Ltd, formerly ICN Biomedicals; catalogue number 3050044) following otherwise the manufacturer's instructions; the other was untreated and kept as a control. After the MRA treatment, the medium was replaced with normal 
A

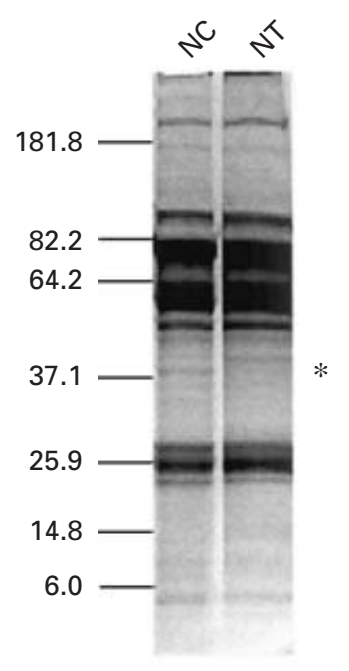

B

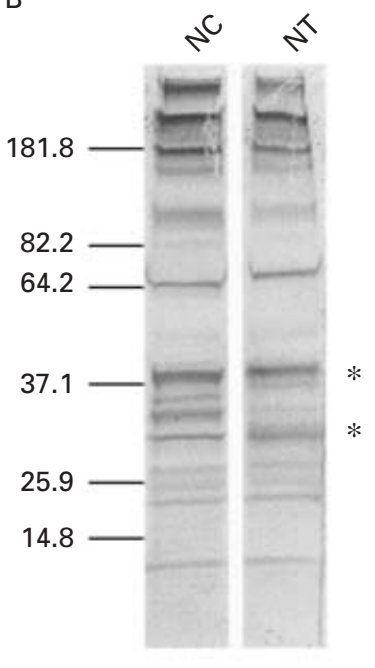

C

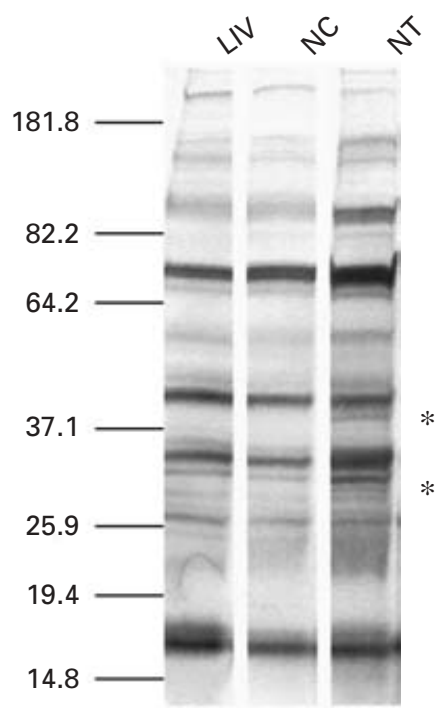

Fig. 1. SDS-PAGE and Western blot analysis of Nc-Nowra tachyzoites. Tachyzoites were either treated with (NT) or without (NC) MRA and were subsequently prepared for SDS-PAGE or Western blotting with a polyclonal mouse antiserum to Neospora caninum. (A) Coomassie blue-stained SDS-PAGE gel of tachyzoite lysates. (B) Western blot analysis of tachyzoite lysates with mouse antiserum to N. caninum. (C) Western blot of ES fraction with mouse antiserum to N. caninum. Liv, NC-Liverpool. * Indicates the proteins and antigens referred to in the text.

RPMI supplemented as above with horse serum and the cultures passaged thereafter in this. Where possible, both control and treated cultures were maintained and passaged at the same frequency at all times, and maintained under similar conditions of culture. All tachyzoite cultures were subsequently analysed as described below, and were passaged no more than 10 times (since treatment) before analysis.

\section{Protein analysis and Western blotting}

Tachyzoites were purified from these cultures by filtration through a $3 \mu \mathrm{m}$ nitrocellulose filter, washed and concentrated by centrifugation at $500 \mathrm{~g}$ in phosphate-buffered saline (PBS), and analysed by SDS-polyacrylamide gel electrophoresis (SDSPAGE) using a Tris-SDS-glycine electrophoresis buffer. Proteins were identified by staining the gels with Coomassie Blue $(0 \cdot 2 \%$ in $50 \%$ methanol and $10 \%$ glacial acetic acid) and then destaining in $7 \%$ glacial acetic acid, 12\% methanol.

Western blotting was performed as follows. After separation by SDS-PAGE, the proteins were transferred by Western blotting onto $0.45 \mu \mathrm{m}$ polyvinylidene fluoride (PVDF) membranes using a standard Tris-glycine buffer. Membranes were blocked for $1 \mathrm{~h}$ at room temperature using PBS containing $0.05 \%$ Tween-20 and $5 \%$ skim milk powder. PVDF strips were then incubated for $1 \mathrm{~h}$ at room temperature with a polyclonal serum to $N$. caninum, which was raised previously in the QS mouse by infection with tachyzoites of Nc-Liverpool (bled 3 weeks postinfection). After 3 washes in PBS containing 0.05\% Tween-20, the strips were incubated for $1 \mathrm{~h}$ at room temperature in blocking buffer containing antimouse IgG conjugated to alkaline phosphatase (Sigma). After 3 further washes, signals were developed using the Sigma fast BCIP/NBT substrate. The reaction was stopped, at a suitable endpoint, by the addition of $3 \%$ trichloroacetic acid.

\section{Production of excreted-secreted (ES) proteins}

Tachyzoites, purified as described above, were resuspended in $1.5 \mathrm{ml}$ of RPMI medium supplemented with $1 \%$ penicillin/streptomycin only (no serum) (Hudson et al., unpublished observations). Tachyzoites were incubated for $3 \mathrm{~h}$ at $37^{\circ} \mathrm{C}$ with mild agitation. Tachyzoites were then centrifuged at $1000 \mathrm{~g}$ for $10 \mathrm{~min}$ at $4{ }^{\circ} \mathrm{C}$ and the supernatants subject to SDS-PAGE analysis.

\section{RESULTS}

An in vitro culture of Mycoplasma-free Nc-Nowra (established by commercial PCR testing) was passaged and 1 of the subcultures was treated with MRA. An identical subculture was not treated and was maintained as a control under otherwise identical conditions.

Purified tachyzoites from both treated and control cultures were analysed by SDS-PAGE and Coomassie blue staining of protein gels (Fig. 1A). Nine abundant proteins were detected, plus a multitude of others. The protein profiles of both populations of tachyzoites were very similar, although several low abundance proteins around the $37 \mathrm{kDa}$ marker varied in abundance between the two. 
Western blotting using mouse serum raised to $N$. caninum by infection of a mouse (day 30 postinfection) was used to probe blots of these tachyzoite extracts (Fig. 1B). The serum detected many proteins. However, the profile of antigens detected between the treated and control tachyzoites differed in the antigens detected between 25 and $37 \mathrm{kDa}$. Tachyzoites that had been treated with MRA were missing several antigens in this size range. The profile of antigens visualized by Western blotting in the control tachyzoite preparations was the same as that identified for other strains of $N$. caninum (e.g. Nc-Liverpool) maintained at UTS over many years.

Analysis of ES antigens produced by the two tachyzoite populations by SDS-PAGE, along with those from a Mycoplasma-free culture of Nc-Liverpool, showed that tachyzoites that had been previously cultured with MRA produce ES antigens that are different in composition from the control tachyzoites. Unlike the control tachyzoites, several more proteins are detectable in MRA-treated tachyzoites. Fig. 1C shows an immunoblot experiment where ES antigens from Nc-Nowra and Nc-Liverpool were probed with a mouse serum raised to $N$. caninum. The ES antigens from treated tachyzoites of Nc-Nowra contained antigens at 30 and $40 \mathrm{kDa}$ that were not present in the ES antigens from untreated tachyzoites.

\section{DISCUSSION}

Mycoplasma are common contaminants of eukaryotic cell cultures, and upon their detection the current recommendations are to either discard the cultures or attempt treatment with an antibiotic. We therefore investigated the effect of MRA treatment upon the composition of tachyzoites of N. caninum. MRA was used in these studies because it is in common use in laboratories for the eradication of Mycoplasma from cultures (Nakai et al. 2000; Uphoff \& Drexler, 2004). The results show clearly that treatment of $N$. caninum with MRA selects for a mutant population of tachyzoites that differs in protein and antigen content from the control cultures. In addition, the ES fraction of treated and untreated tachyzoites differs, thereby indicating the existence of potential differences in their basic secretory pathways. These results were so dramatic that no further studies were performed in order to ascertain the suitability of MRA for removal of Mycoplasma from cultures of N. caninum.

The Mycoplasma Removal Agent used in this study was a 4-oxo-quinolone-3-carboxylic acid derivative, whose mode of action is really unknown. Fluoroquinolones are broad-spectrum antibiotics, and some of these are recognized as having antiToxoplasma and anti-malarial activity (Khan et al. 1996, 1999). Studies on Mycoplasma have shown that fluoroquinolones inhibit DNA gyrase and topoisomerase IV activities and that mutations in these gene loci correlate with the development of resistance to this drug (Bebear et al. 2000, 2003). Research performed with $T$. gondii has shown that ciprofloxacin, when used at high concentrations, inhibits replication of the apicoplast, thereby preventing tachyzoite replication which results in cell death (Fichera \& Roos, 1997). There was therefore good reason to be cautious about the use of MRA for the eradication of Mycoplasma from $N$. caninum cultures.

In summary, we do not support the use of antibiotics for the removal of Mycoplasma from cultures of $N$. caninum (or Toxoplasma gondii). Where cultures are contaminated, the best course of action is to discard them. However, infection of a laboratory animal and re-isolation of the parasite from infected tissues may also be an appropriate course of action if cultures are particularly valuable. On a more positive note, the mutagenesis of $N$. caninum using MRA may provide novel strains with which to investigate the biology of this interesting parasite.

\section{REFERENCES}

Bebear, C. M., GRAU, O., Charron, A., Renaudin, H., GRUSON, D. \& BEBEAR, C. (2000). Cloning and nucleotide sequence of the DNA gyrase (gyrA) gene from Mycoplasma hominis and characterization of quinoloneresistant mutants selected in vitro with trovafloxacin. Antimicrobial Agents and Chemotherapy 44, 2719-2727.

BEbEAR, C. M., RENAUdin, H., CHARRON, A., ClERC, M., PEREYRE, s. \& BEBEAR, C. (2003). DNA gyrase and topoisomerase IV mutations in clinical isolates of Ureaplasma spp. and Mycoplasma hominis resistant to fluoroquinolones. Antimicrobial Agents and Chemotherapy 47, 3323-3325.

FICHERA, M. E. \& ROOS, D. S. (1997). A plastid organelle as a drug target in apicomplexan parasites. Nature, London 390, 407-409.

HENSMANn, M. \& KWIATKOWSki, D. (2001). Cellular basis of early cytokine response to Plasmodium falciparum. Infection and Immunity 69, 2364-2371.

KHAN, A. A., ARAUJO, F. G., BRIGHTY, K. E., GOOTZ, T. D. \& Remington, J. S. (1999). Anti-Toxoplasma gondii activities and structure-activity relationships of novel fluoroquinolones related to trovafloxacin. Antimicrobial Agents and Chemotherapy 43, 1783-1787.

KHAN, A. A., SLIFER, T., ARAUJO, F. G. \& REMington, J. S. (1996). Trovafloxacin is active against Toxoplasma gondii. Antimicrobial Agents and Chemotherapy 40, 1855-1859.

MILlER, C. M. D., QUINN, H. E., WINDSOR, P. A. W. \& ELLIS, J. T. (2002). Characterisation of the first Australian isolate of bovine Neospora caninum. Australian Veterinary Fournal 80, 620-625.

misaWa, s., aoshima, M., Takaku, H., matsumoto, M. \& HAYASHI, H. (1994). High-level expression of Mycoplasma arginine deiminase in Escherichia coli and its efficient renaturation as an anti-tumor enzyme. Fournal of Biotechnology 36, 145-155. 
NAKAI, N., KAWAGUCHI, C., NAWA, K., KOBAYASHi, S., Katsuta, Y. \& WATANabe, M. (2000). Detection and elimination of contaminating microorganisms in transplantable tumors and cell lines. Experimental Animals 49, 309-313.

RAPPElli, P., CARTA, F., DElOGU, G., ADDIS, M. F., DESSi, D., CAPPUCCINElli, P. \& FIORI, P. L. (2001). Mycoplasma hominis and Trichomonas vaginalis symbiosis: multiplicity of infection and transmissibility of M. hominis to human cells. Archives of Microbiology 175, $70-74$.

ROWE, J. A., SCRAGG, I. G., KWIATKOWSKi, D., FERGUSON, D. J., CARUCCI, D. J. \& NEWBOLD, C. I. (1998).

Implications of Mycoplasma contamination in
Plasmodium falciparum cultures and methods for its detection and eradication. Molecular and Biochemical Parasitology 92, 177-180.

TURrini, F., Giribaldi, G., VAlEnte, E. \& AREse, P. (1997). Mycoplasma contamination of Plasmodium cultures - A case of parasite parasitism. Parasitology Today 1997, 367-368.

UPHofF, C. C. \& DREXler, H. G. (2002). Comparative antibiotic eradication of Mycoplasma infections from continuous cell lines. In Vitro Cellular and Developmental Biology. Animal 38, 86-89.

UPHOFF, C. C. \& DREXLER, H. G. (2004). Elimination of Mycoplasma from infected cell lines using antibiotics. Methods in Molecular Medicine 88, 327-334. 